\title{
Helicobacter Infection and Chronic Liver Diseases
}

\author{
Zhao-chun Chi, Xin-juan Yu and Quan-jiang Dong
}

This paper reviews the recent Helicobacter infection associated with chronic liver disease. The bacteriology, prevalence, pathogenesis and diagnosis were reviewed. Future work should be conducted on the pathogenesis and treatment of this disease.

Key words: Helicobacter pylori; H. hepaticus; Chronic liver diseases

$\mathrm{I}$ recent years, the research reports on Helicobacter pylori (H. pylori) infection and chronic liver disease are increasing. So far, more than 20 kinds of Helicobacter species have been found, among which $H$. pylori is the most important one for its strong pathogenicity to human and wide popularity. Many studies have pointed out that H. pylori is closely related to the incidence and prognosis of chronic hepatitis, liver cirrhosis and hepatocellular carcinoma. It is mainly caused by inflammation and immune mechanisms. This paper is a review on recent years' researches about the association between $H$. pylori and chronic liver diseases.

\section{ETIOLOGY AND BACTERIOLOGY}

Helicobacterium $\mathrm{h} 1 \mathrm{~s}$ been found to contain more than 20 species up to now. Three species take human as the major host, namely $H$. pylori, Helicobacter heilmanni (H. heilmanni) and Helicobacter cinaedi (H. cinaedi). Other species take animal as the host, such as ferrets, pigs, dogs, cats, rats, mice, terns and so on. Among all the species, H. pylori is the most important pathogene with serious pathogenicity and widespread epidemics, which can cause gastrointestinal and parenteral diseases, such as chronic gastritis, peptic ulcer, gastric cancer, liver diseases, cardiovascular and cerebrovascular diseases, sepsis, pneumonia, bronchitis, anemia, tumor, ear nose throat diseases and skin diseases, etc.

Department of Gastroenterology, Qindao Municipal Hospital, Affiliated Hospital of Ocean University of China, Qingdao 266071, China Correspondence:Zhao-chun Chi, E-mail:c.z.chow@163.com
Helicobacter hepaticus $(\mathrm{Hh})$ is a new type of Helicobacter associated with H. pylori, which can cause chronic hepatitis, and even lead to liver cancer in mice. Whether the Hh is the same with species separated from the mice has not been determined.

There are detailed study and much reports on bacteriology of H. pylori. Hh is a kind of Gramnegative spiral-shaped bacterium with 1-3 turns. It has different sizes, with length of $1.55 \mu \mathrm{m}$, width of 0.2 $0.3 \mu \mathrm{m}$. Observed in electron microscope, the surface of $\mathrm{Hh}$ is smooth, and there is one scabbard flagella at each end of the bacteria, which make the bacteria have function of movement.

$\mathrm{Hh}$ is similar to $H$. pylori, which can produce urease, peroxidase, oxidase, reduction of nitride to nitrate, produce hydrogen sulfide $\left(\mathrm{H}_{2} \mathrm{~S}\right)$ and hydrolyze indoleacetic acid. Hh requires high nutrition, so they are often inoculated in Columbia medium with $7 \%$ sheep blood, $10 \mathrm{~g} / \mathrm{L}$ vancomycin, $2 \mathrm{~g} / \mathrm{L}$ amphotericin $\mathrm{B}$ and $2.5 \mathrm{~g} / \mathrm{L}$ polymyxin $\mathrm{B}$. Cultured for 3-7 days at the microaerophilic environment (i.e. $85 \% \mathrm{~N}_{2}, 10 \%$ $\mathrm{CO}_{2}, 5 \% \mathrm{O}_{2}$ ) with high humidity and the condition of $37{ }^{\circ} \mathrm{C}$, transparent microcolonies with a diameter of $1-2 \mathrm{~mm}$ will be observed, and there is no obvious hemolysis. ${ }^{1}$

\section{HELICOBACTER PYLORI AND CHRONIC LIVER DISEASES}

\section{The mechanism of $\mathrm{H}$. pylori combined with} chronic liver diseases

The studies mainly focused on $H$. pylori and cirrhosis. 
The defensive capability of the patients with cirrhosis to infection is reduced, which can cause endotoxemia and $H$. pylori proliferation in gastric mucosa, and $H$. pylori can also reach the liver through the bloodstream and cause hepatitis. The incidence of peptic ulcer, gastritis, especially atrophic gastritis in patients with chronic liver diseases is higher than that of non liver disease group. The occurrence of the above pathology in chronic liver diseases has important and direct relationship with $H$. pylori infection. On the contrary, reports about effects of $H$. pylori on liver diseases is rare. However, cytokines release of hepatocytes induced by virulence factors of $H$. pylori, immune response (cellular and humoral immunity) to $H$. pylori and gene mutation induced by $H$. pylori can lead to hepatocytes damage, then result in liver diseases. ${ }^{2}$ But it needs further study to clarify its pathogenesis and pathophysiological changes.

H. pylori infection can induce many extragastric disease, including cirrhosis and hepatocellular carcinoma, but the mechanism of $H$. pylori inducing liver damage is poorly understood. Le et al studied on the different state of physiology and pathology of liver in vitro in mouse primary hepatocytes cultured in test tubes, and found that primary hepatocytes could assemble actin-based cytoskeletal structures called podosomes at the ventral plasma membrane, while $H$. pylori infection further stimulated the formation of podosomes, and TGF- $\beta$ regulated podosome formation in endothelial cells. ${ }^{3}$ The same results were obtained with the hepatoma cell line Huh7. Podosome formation could degrade the capacities of hepatocytes and reduce cell motility. Therefore, podosome assembly may result in hepatocyte malfunction.

H. pylori prevalence of peptic ulcer is more frequent in cirrhotic patients than controls. Zullo et al evaluated wether cirrhotic patients had an increased bacterial density and/or a higher prevalence of $H$. pylori cagA-positive strains with a casecontrol study. ${ }^{4} H$. pylori infection was detected by Giemsa staining, bacterial density was assessed using difference over baseline (DOB) values $\left({ }^{13} \mathrm{C}\right.$ urea breath test), and cagA status was established at serology level. The results showed that both DOB values and cagA prevalence had no significant differences between cirrhotic patients and controls. However, sero-prevalence of $H$. pylori in cirrhotic patients with hepatic encephalopathy was significantly higher than that of cirrhotic patients wihtout hepatic encephalopathy ( $51.61 \%$ vs $48.39 \%)$, but the clinical and biochemical parameters were similar between the two group. H. pylori infection may be not the independent risk factor of hepatic encephalopathy in patients with cirrhosis.

\section{H. pylori infection and liver cirrhosis combined with peptic ulcer}

The incidence of chronic liver diseases combined with peptic ulcer was $10 \%-25 \%$. The incidence of liver cirrhosis combined with gastric ulcer was $12.2 \%$, while that of liver cirrhosis, hepatocellular carcinoma combined with gastric ulcer was $25.8 \%$. Villalan et al studied on the liver cirrhosis combined with peptic ulcer. The patients were divided into three groups: (A) liver cirrhosis with peptic ulcer (PUD); (B) uncomplicated PUD; (C) simple liver cirrhosis without PUD. ${ }^{5}$ Results showed that cirrhotic patients with PUD had a significantly lower prevalence of H. pylori compared to uncomplicated PUD (46.9\% vs $80 \%, P=0.04)$. While the $H$. pylori eradication rate was similar between cirrhotic and non cirrhotic patients, but the ulcer healing rate of cirrhotic patients was significantly lower than that of non-cirrhotic patients $(48 \%$ vs $8 \%)$. H. pylori eradication could not decrease the residual ratio of ulcers. This indicated that $H$. pylori infection might not be a major risk factor for PUD in cirrhotic patients. Hence, routine H. pylori eradication might not be warranted in patients with cirrhosis and pepotic ulcer disease. On the relationship between $H$. pylori infection and gastric acid $\mathrm{pH}$ in patients with liver cirrhosis, Nam et al reported 29 cases of liver cirrhosis and 33 cases of healthy controls. ${ }^{6}$ Among them, 12 cases had hepatitis B with liver cirrhosis, 12 cases had alcoholic liver cirrhosis, 5 cases had hepatitis $\mathrm{C}$ with cirrhosis. The gastric $\mathrm{pH}$ of patients with liver cirrhosis was higher than that of controls and a larger proportion of cirrhotic patients have hypochlorhydria. The prevalence of $H$. pylori in liver cirrhosis patients was 
similar to that in cointrols and no correlation was found between gastric and blood $\mathrm{NH}_{3}$ levels. Thus, $H$. pylori infection does not seem to play a major role in generation of elevated $\mathrm{NH} 3$ associated with hepatic encephalopathy.

Taiwan scholar Lo et al found that the prevalence of $H$. pylori in 104 patients with cirrhosis and duodenal ulcers was $52 \% .^{7}$ Recurrent duodenal ulcer within 1 year were noted in 21 of 36 patients (58\%) who achieved H. pylori eradication. Recurrent duodenal ulcers also were noted 8 of the 18 patients (44\%) who remained $H$. pylori positive. Eradication of $H$. pylori in patients with cirrhosis and duodenal ulcers did not effectively reduce the recurrence of ulcers.

Wyszomirska et $\mathrm{al}^{8}$ studied on 46 patients with chronic liver diseases and 27 dyspeptic patients. A gastroduodenal lesions were found in $38(82.6 \%)$ patients with liver diseases and it was significantly more frequent than that among controls. H. pylori infection was detected by histological assessment in $13(28.2 \%)$ patients with chronic liver diseases and in 17 (62.9\%) controls. Patients with chronic liver diseases showed higher risk of developing gastroduodenal lesions regardless of the presence of the $H$. pylori infection.

The prevalence rate of $H$. pylori in patients with liver cirrhosis was different according to different reports. Some reports indicated chronic hepatitis $\mathrm{C}$ with cirrhosis was associated with $H$. pylori, and the prevalence of $H$. pylori in chronic hepatitic $\mathrm{C}$ with cirrhosis was higher than that without cirrhosis. Quciroz et al studied on 106 cases of chronic hepatitis C, among which, 47 cases (44.3\%) had cirrhosis, 33/47 (70.2\%) was H. pylori positive; 59 cases had no cirrhosis, only 28 cases $(47.5 \%)$ was $H$. pylori positive. ${ }^{9}$ But Urso et al reported that the prevalence rate of $\mathrm{H}$. pylori in 109 cases of chronic hepatitis $\mathrm{C}$ with cirrhosis was $23.8 \%$, which indicated that the prevalence rate of $\mathrm{H}$. pylori in chronic hepatitis $\mathrm{C}$ with cirrhosis was low. ${ }^{10}$ While a Chinese report showed that prevalence rate of $H$. pylori in 457 cases of liver cirrhosis was up to $60.6 \%$. The numbers varied widely.

Stalke et al studied on 62 cases of patients with chronic liver disease by DGGE and sequence analysis to identify $H$. pylori, 60/62 (97\%) and 25/25 (100\%) of the Helicobacter genus-positive gastric and liver samples were identified, respectively, whereas DNA of $H$. heilmannii was detected in 2/62 (3\%) of the Helicobacter genus-positive samples. H. pylori cagA gene was detected in 23/62 (36\%) and 3/25 (12\%) gastric and liver tissue samples, respectively. H. pylori-like antigens were detected in 61/97 (63\%) gastric mucosa and in 40/97 (41\%) liver tissue samples. The prevalence of the cagA gene was higher in gastric samples compared with that in liver samples, which suggested a possible role of cagA-negative $H$. pylori-like organisms in chronic liver diseases. No significant correlation was found between the presence of $H$. pylori-like DNA and antigen in the liver and liver function tests. ${ }^{11}$

\section{H. pylori and chronic virus hepatitis}

Wang et al studied on the prevalence rate of $H$. pylori in $235 \mathrm{HBV}$ carriers (ASCs), 1637 normal adults and 573 alcohol users. ${ }^{12}$ In 235 cases of ASCs, H. pylori prevalence rate was $38.67 \%$, which has no significant differences compared with that in 1637 nomal adults $(35.94 \%, P=0.352)$. H. pylori prevalence rate $(26.98 \%)$ was significantly decreased in alcohol users with normal liver function compared with that in the normal adults $(P=0.014)$. H. pylori prevalence rate was increased to $35.20 \%$ in alcohol users with abnormal liver function, which was significantly different from that of alcohol users with normal liver function $(P=0.042)$. The results indicated that the H. pylori prevalence rates of ASCs and normal adults were similar, and moderate alcohol consumption may facilitate elimination of $H$. pylori, while $H$. pylori infection is increased in alcohol users with abnormal liver function.

Patients with chronic hepatitis $\mathrm{C}$ virus infection may progress to fibrosis, cirrhosis, liver failure or liver cancer. $H$. pylori infection exists in the stomach of $50 \%$ of the worldwide population. H. pylori DNA has been identified in the human livers and has been complicated with chronic liver disease and liver cancer. Sakr et al studied on the histological and histochemical changes of HCV patients with or 
without $H$. pylori infection, and pointed out that the HCV patients coinfected with H. pylori had higher NIC scores and had pronounced fibrosis stages than $\mathrm{HCV}$ patients without $H$. pylori infection. ${ }^{2}$ Glycogen and total proteins decrease was marked in liver of HCV patients coinfected with H. pylori. This is the evidence that $H$. pylori can aggravate liver injury.

\section{H. pylori and hepatocellular carcinoma}

Many reports pointed out that $H$. pylori infection can cause hepatitis, liver cirrhosis and hepatocellular carcinoma, but most of these reports are summary of clinical data and animal model studies. So far the pathogenesis of HCC caused by H. pylori remains unclear. Prevalence rate of Helicobacter in liver tissue of patients with $\mathrm{HCC}$ was $10.7 \%$.

Studies show that HCC patients have a higher prevalence rate of $H$. pylori than other chronic liver disease (CLD) and healthy poputation. Chen et al detected $H$. pylori in $30 \mathrm{HCC}$ patients, and found that 1 case $(3 \%)$ was $H$. pylori positive with urease tests, 9 cases $(30 \%)$ were $H$. pylori positive with immuohistochemical staining, and 18 cases $(60 \%)$ were $H$. pylori positive with PCR to detect $H$. pylori $16 \mathrm{~S}$ rRNA, but all of the controls and CLD cases were $H$. pylori negative using the three methods. ${ }^{13}$ Sequencing analysis of $H$. pylori $16 \mathrm{~S}$ rRNA in liver tissue showed a $98.8 \%$ homology with the $H$. pylori $16 \mathrm{~S}$ rRNA gene. A greater incidence of $H$. pylori infection in the liver is identified in patients with liver cancer than in healthy subjects and CLD patients, suggesting a potential correlation between $H$. pylori infection to liver cancer. Another study showed that 30 cases $(65.2 \%)$ in 46 cases of $\mathrm{HCC}$ were serum $H$. pylori positive, and the $H$. pylori positive rate was higher than that of the controls [ 25/46 (54\%)].

\section{H. pylori and autoimmune diseases}

Most scholars think Helicobacter infection and autoimmune liver diseases have low correlation. Dzierzanowska-Fangrat et al found no correlation between $H$. pylori infection and autoimmune hepatitis in children. ${ }^{14} \mathrm{H}$. pylori antibody prevalence rate between patients with autoimmune hepatitis (AIH) and the controls also had no significant differences (22\% vs 14\%). Another study found H. pylori seroprevalence increased in autoimmune liver diseases. The results showed that $H$. pullorum, Helicobacter Bilis and $\mathrm{Hh}$ positive rates were 30\%, $22 \%$ and $22 \%$ in primary sclerosing cholangitis (PSC) patients with dot immunobinding assay, respectively. Seroprevalence was significantly higher in patients with chronic autoimmune liver diseases than in healthy blood donors $(P<0.001)$. Sustained liver cell necrosis is characteristic of autoimmune hepatitis, often progresses to cirrhosis. A case-control study found that $H$. pylori prevalence rate of patients with chronic autoimmune hepatitis and blood donors had no significant differences $(64.5 \%$ vs $53.2 \%, P=0.3)$.

\section{Hh and liver diseases}

Studies on relationship between $\mathrm{Hh}$ and liver are mostly carried in animal models. Uninfected mice took Hh standard strains of ATCC51448 orally, then scattered liver cell necrosis appeared in rat liver after 2 weeks. The necrosis aggravated at week 1016, and reached the peak at week 28. One mouse had hepatoma. Another study found that liver cells of $\mathrm{A} / \mathrm{J} \mathrm{Cr}$ mice infected with $\mathrm{Hh}$ gradually increased in size, oval cell proliferated, and the focal nodular hyperplasia formated after 1 year. Pathological changes from chronic hepatitis to liver cancer were observed at week 78. Different Hh strains can cause different degrees of hepatitis because of different genetic composition changes. The $\mathrm{A} 70 \mathrm{~Kb}$ gene (HHGI1) island in strain ATCC 51449 is a pathogenic island (PAI). In order to determine the role of PAI in vivo, Boutin et al administrated Hh3B1 strains, one of the three inoculated Hh strains to mice, which contains the complete PAI, and strains HhNET and HhG lack all or large parts of the HHGI1, respectively. ${ }^{15}$ Mice infected with $\mathrm{HhG}$ and HhNET developed less-severe hepatitis than the male A/J Cr mice infected with Hh3B1.

$\mathrm{Hh}$ is considered as one of the causes of chronic hepatitis and hepatocellular carcinoma in mice. Pathogenesis of $\mathrm{Hh}$ includes host factors, oxidative damage, oncogene activation, and the abnormal expression of cyclins. With the aggravation of pathological changes in liver after Hh infection, 
8-hydroxy deoxyguanosine (8-OXO-dG) significantly increased, which is a sensitive marker of oxidative damage. Using immunohistochemistry and Western blot to detect the liver pathological changes at different stages in $\mathrm{A} / \mathrm{JCr}$ mice infected with $\mathrm{Hh}$, researchers found that EGF, TGF- $\alpha$ increased in the early stage of disease, and were 8 times and 9 times of the control group, respectively. EGF significantly increased in tumor tissues, while cyclin D1 and CDK4 increased significantly in all the infected liver tissue. Their expression were 8 times and 50 times at 18 months, respectively, but the expression was highest in liver tumors. These changes suggested that the combined action of growth factor, cell cycle proteins, transcription factors play an important role in the pathogenesis of Hh related liver tumor. ${ }^{1}$

In recent years, many scholars found that hepatitis $\mathrm{C}$ and Hh had a synergistic effect, which cause increased incidence of hepatocellular carcinoma. Hh can increase the liver injury. The presence of carcinoma is more likely to occur on a background of hepatitis C. Researches found that the effect of the mouse genetic background was greater than that of the HCV transgene, and the synergistic effect between $\mathrm{HCV}$ and Hh participated in hepatocellular carcinoma. ${ }^{16}$

Hh promoting tumorigenesis is accompanied by increased bile acids and immune response. Androstane receptor (CAR) is a nuclear receptor that regulates enzymes involved in endobiotic and xenobiotic metabolism. CAR activation is a mechanism of xenobiotic tumor promotion. CAR knockout (KO) and wild-type (WT) male mice were treated with or without the tumor intiator diethylnitrosamine (DEN) at 5 weeks of age, and then orally inoculated with $\mathrm{Hh}$ or sterile media at 8 weeks of age. At approximately week 50 postinoculation, mice were euthanized for histopathologic, microbiological, molecular, and metabolic analyses ${ }^{17}$. Hh infection induced hepatitis in WT and KO mice with or without DEN that correlated with significant upregulation of TNF- $\alpha$ and toll receptor Tlr2. DEN-treated Hhinfected $\mathrm{KO}$ mice exhibited increased numbers of liver lobes with dysplasia and neoplasia and increased multiplicity of neoplasia. Enhanced tumor promotion was associated with decreased hepatic expression of P450 enzymes Cyp2b10 and Cyp3a11, and increased serum concentrations of chenodeoxycholic acid. The results suggested a close relationship between $\mathrm{Hh}$ infection and metabolic detoxification of endobiotics and persistent microbial-induced immune response, which cause the impairment and tumorigenesis of hepatocytes.

To sum up, there is an association between $\mathrm{Hh}$ infection with hepatitis, liver cirrhosis and liver cancer. The possible mechanism of liver disease caused by Hh: (A) autoimmune response: Hh and heat shock protein 70 (HSP70) has common cross antigen, suggesting that autoimmunity may play a role in the pathopoiesia of Hh. (B) Oxidative damage: with the progression of disease, $8-\mathrm{OXO}-\mathrm{dG}$ increases significantly, and causes the ring pyrimidine 6-hydroxy pyrimidine N-1, N (2) MDA-deoxyguanosine (MIdG) formation, leading cell mutation and finally inducing carcinoma. (C) Oncogene mutations: Hh activates oncogene mutations and leads to cancer, but the detailed mechanism is still unclear. (D) Cyclin D1 overexpression: about $10 \%$ of the liver cancer show high cyclin D1. Hh causes an abnormal proliferation of liver cells, finally leads to cancer.

\section{PROSPECT}

Helicobacter, especially $H$. pylori and Hh being associated with chronic liver disease has been achieved consensus. In recent years, case-control studies and animal models on the association of the Helicobacter and chronic liver diseases gradually increased. China is a liver disease prone country, and $H$. pylori high prevalence areas. Therefore, there's urgent need to strengthen the study on the role of inflammatory factors, immune factors, clinical pathology and molecular biology on the pathogenesis of chronic liver disease with Helicobacter infection, and clarify the pathogenesis and epidemiology, to reduce the incidence of chronic liver diseases and improve the diagnosis, treatment and prognosis of Helicobacter induced liver diseases. 


\section{REFERENCES}

1. Chi ZC. Helicobacter pylori infection and chronic liver disease. Practical clinical hepatology. China, People's Medical Publishing House, 2014.

2. Sark SA, Badrah GA, Sheir RA. Histological and histochemical alterations in liver of chronic hepatitis patients with Helicobacter pylori infection. Biomed Pharmacother 2013;67(5):367-374.

3. Le Roux-Goglin E, Varon C, Spuul P, Asencio C, Mégraud F, Génot E. Helicobacter infection induces podosome assembly in primary hepatocytes in vitro. Eur J Cell Biol 2012;91(3):161170 .

4. Zullo A, Sanchez-Mete L, Hassan C, Diana F, Festuccia F, Attili AF, et al. Helicobacter pylori density and CagA status in cirrhosis patients: a case-control study. J Gastroenterol Hepatol 2004;19(10):1174-1178.

5. Villalan R, Maroju NK, Kate V, Ananthakrishnan N. Helicobacter pylori eradication indicated in cirrhotic patients with peptic ulcer disease. Gastroenterol 2006;27(4):166-168.

6. Nam YJ, Kim SJ, Shin WC, Lee JH, Choi WC, Kim KY, et al. Gastric $\mathrm{pH}$ and Helicobacter pylori diagnosis in patients with liver cirrhosis. Korean J Hepatol 2004;10(3):216-212.

7. Lo GH, Yu HC, Chan YC, Chen WC, Hsu PI, Lin CK, et al. The effects of eradication of Helicobacter pylori on the recurrence of duodenal ulcers in patients with cirrhosis. Gastrointest Endosc 2005;62(3):350-356.

8. Wyszomirska RM, Ribeiro LT, Monteiro JS, Vidal BP, Melo RM, Miranda CT. Association between Helicobacter pylori infection and gastroduodenal lesions in patients with chronic liver disease. Arg Gastroenterol 2009;46(4):256-260.

9. Queiroz DM, Rocha AM, Rocha GA, Cinque SM, Oliveira AG, Godoy A, et al. Association between Helicobacter pylori infection and cirrhosis in patients with chronic hepatitis $\mathrm{C}$ virus. Dig Dis Sci 2006;51(2):370-373.

10. Urso G, Interlandi D, Puglisi M, Abate G, Bertino G, Raciti C, et al. Role of Helicobacter pylori in patients with portal hypertensi gastropathy by liver cirrhosis hepatitis $\mathrm{C}$ virus- related. Minerva Gastroenterol Dietol 2006;52(3):303-308.

11. Stalke P, Al-Soud WA, Bielawski KP, Bakowska A, Trocha H, Stepinski J, et al. Detection of Helicobacter species in liver and stomach tissues patients with chronic liver disease using polymerase chain reaction-denaturing gradient gel electrophoresis and immunohistochemistry. Scand J Gastroenterol 2005;40(9):1032-1041.

12. Wang MY, Yue JY, Zhang YX, Liu XD, Gao XZ. Helicobacter pylori infection in asymptomatic HBV carriers, alcohol users and normal adult population in Shandong province, China. Clin Res Hepatol Gastroenterol 2011;35(8-9):560-562.

13. Chen R, Chen XP, Lin QQ, Lin BL, Cao HJ. Detection of Helicobacter species related genes coding for 16S Rrna in the liver tissue of patients with chronic liver diseasel. Nan Fang Yi Ke Da Xue Xue Bao 2010;30(1):131-132.

14. Dzierzanowska-Fangrat K, Nilsson I, Wozniak M, Jozwiak P, Rozynek E, Woynarowski M, et al. Lack of an association between Helicobacter infection and autoimmune hepatitis in children. Pol J Microbiol 2006;55(2):157-159.

15. Boutin SR, Shen Z, Rogers AB, Feng Y, Ge Z, Xu S, et al. Different Helicobacter hepaticus strains with variable genoma content induce various degrees of hepatitis. Infect Immun 2005; 73(12):8449-8452.

16. Le Roux-Goglin E, Dubus P, Asencio C, Jutand MA, Rosenbaum J, Mégraud F. Hepatic lesions observed in hepatitis $\mathrm{C}$ virus transgenic mice infected by Helicobacter hepaticus. Helicobacter 2013;18(1):33-40.

17. García A, Zeng Y, Muthupalani S, Ge Z, Potter A, Mobley MW, et al. Helicobacter hepaticus-induced liver tumor promotion is associated with increased serum bile acid and a persistent microbial-induced immune responses. Cancer Res 2011; 71(7):2529-2540. 